http://dx.doi.org/10.21611/qirt.2014.205

\title{
Thermography in laser ablation control
}

\author{
by V.Veikutis*, A. Sakalauskaite ${ }^{\star \star}$, K. Stasiukynaite ${ }^{\star \star}$ \\ * Lithuanian University of Health Sciences, Institute of Cardiology, Kaunas, Lithuania, \\ vincentas.veikutis@med.kmu.It \\ ** Lithuanian University of Health Sciences, Kaunas, Lithuania, agne.sakalauskaite@ymail.com; \\ stasiukynaite@gmail.com
}

\begin{abstract}
The aim of the study was to estimate destruction effect to different heart structures using laser methodology in animal (pig) model.

Ablation procedure was performed on right and left pulmonary veins area inserting electrode through a. femoralis or incision in right/left auricle. The effects of ablation were observed visually and temperature controlled by thermography camera P640 (FLIR Systems, USA).

Using standard recommended parameters $40 \mathrm{~W} / 180 \mathrm{sec}$ and $35 \mathrm{~W} / 120 \mathrm{sec}$ evoked quick critical scar formation. Temperature in cardiac surface differs from 34.5 till $70.2^{\circ} \mathrm{C}$ and directly depends from electrode compression level and energy power. Optimal detected ablation parameters $-20-30 \mathrm{~W} / 120$ sec.

\section{Background}

Recently most of the cardiac rhythm disorders are corrected by transvenous way, using radiofrequency, microwave, laser energies or cryoablation. In over then 20 years of clinical experience, catheter radiofrequency ablation (RFA) has been standard and mostly developed approach. Unfortunately, despite of increased experience and using modern RFA technologies short- and long-range postoperative complications and relapses fluctuate from 0,5 to 14 percent $^{[1,2,3]}$. Usage of cryoenergy is high effective, especially in atrial fibrillation correction procedures in pulmonary veins zone, but technically complicated and relatively expensive. Microwave weren't useful in clinical practice because of huge thrombogenesis, uneven endocardial lesions and high perforation rate ${ }^{[4]}$. In the $1980 \mathrm{~s}$, were developed and tested successfully laser ablation methodology ${ }^{[5,5]}$. The results showed that by using an open-irrigated laser catheter system, deep lesions can be achieved within seconds without thrombus formation or steam pop ${ }^{[7]}$. Recently, most novel techniques are optimized and tested this catheter system in an isolated thigh-muscle model. Aim of our study was to test laser ablation technique doing circular laser ablation procedure in right-left upper pulmonary vein area, analyze possible mechanisms of complications, measure and determine optimal ablation parameters on beating pig heart model, comparing results with RFT and cryo-ablation results.
\end{abstract}

\section{Methods}

12 female pigs (weight $~ 20-25 \mathrm{~kg}$ ) used in experimental study. Hearts were exposed via sternotomy and pericardtomy, destruction of various heart areas was performed from the endocardial surface by inserting the electrode through femoral vein or the incision on the right or left auricle. For RFA standard 4 and $8 \mathrm{~mm}$ „Biosense Webster"(Johnson\&Johnson,USA) intracardial electrodes were used. Destruction was performed using Prucka Engineering ${ }^{\circledR}$ RF energy generator OSYPKA HAT 200S. Destruction parameters: energy - 10-50 W, time - 10-60 sec. Temperature changes in epicardial surface registered with thermo camera ThermaCAM P640, emission $\dot{\varepsilon}-0.98$, temperature sensitivity $\leq 0.05^{\circ} \mathrm{C}$. After destruction, damaged zones were excised and evaluated histological. Three excised hearts were given for anatomorphological analysis - intracardial nervous tracts topography and state identification. Cryodestruction was performed using cryoaplicator "Cryocauter KCH 450". Cryodestruction parameters: atrials - application time $1 \mathrm{~min}$. and temperature $-80^{\circ} \mathrm{C}$. For laser ablation we used loop form 10F electrode-laser catheter, which was inserted pervenously through the a. femoralis, position in the heart controlled by finger, ablation parameters - 20-35 W, 60 s. Temperature changes in effected heart epicardial surface were registered with thermocamera ThermaCAM P640 (FLIR Systems). During the procedures changes in tissues were observed visually, later destruction zones were excised, cute and the damage of the myocardium was evaluated histological.

\section{Results}

During RFA procedures on surface of atriums, destructive thermo effect $\left(>46^{\circ} \mathrm{C}\right)$ was observed after $5-7 \mathrm{~s}$, after application, despite of power of energy and type of electrode. Dotted zone of total destruction using $20-30 \mathrm{~W}$ power and both type electrodes was $3-4 \mathrm{~mm}$, whereas using $50 \mathrm{~W}-4-6 \mathrm{~mm}$. Partial destruction zone usually was equal or marginally, but regularly major than total destruction zone (pic.1A). Usually zone of destruction was ellipsoid, whereas temperature restoration occurred in $25-78 \mathrm{~s}$., subject to power of energy, time of application and type of electrode. Peculiarities of RFA in site of pulmonary veins were similar comparing with free wall of atrium, however damage area was 20 - 30 percent greater and destruction site was like irregular ellipse pointed toward pulmonary veins flow to atrium. (pic.1B). 30 and $50 \mathrm{~W} 20-60$ s applications outcomes were similar in total damage site, but partial damage sites differs significantly according to power and time of application. 
A

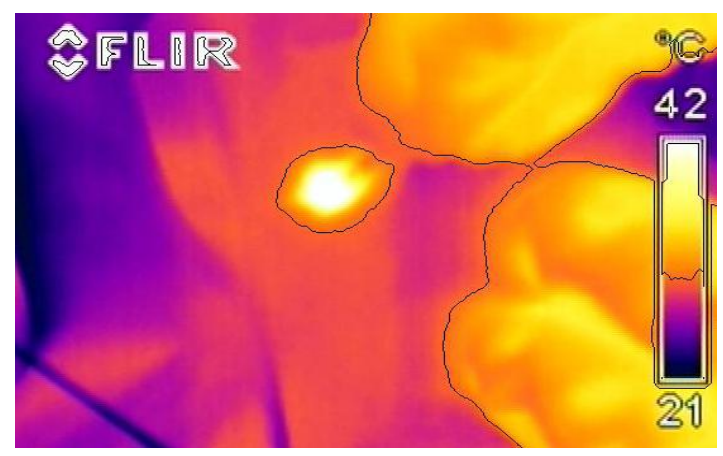

B

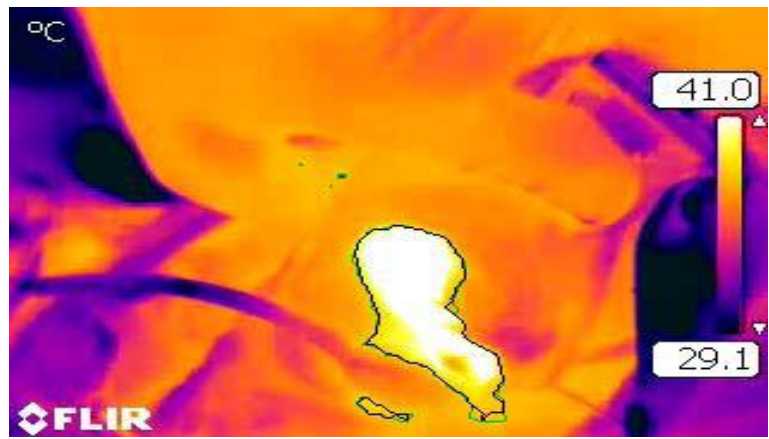

Pic.1. A - total and partial (marked) destruction zones in site of pulmonary veins ostium, using 20 s. $30 \mathrm{~W}$ power; B the same, just using 20 s. 50 W power.

Using cryoablation, destruction zone was total, even and forming regular oval. Destruction zone was statistically reliably even in all sites of heart and almost independent from application time. The same we observed using laser ablation. The laser causes localized heating-up of the heart tissue, with temperature scale $34.1-70.2^{\circ} \mathrm{C}$ and enough restricted scar formation (pic.2).

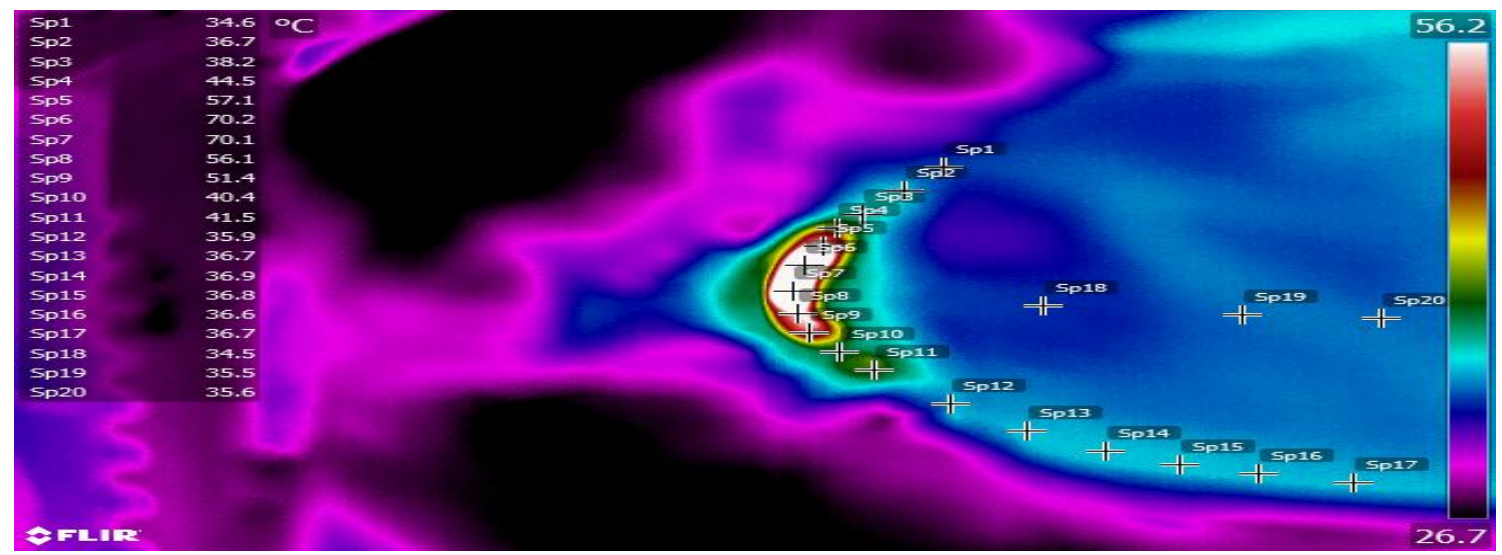

Pic.2. Thermogramm of laser destruction in site of pulmonary veins ostium, using $60 \mathrm{~s}$ and $35 \mathrm{~W}$ power.

\section{Conclusions}

Usage of thermography enable to estimate peculiarities and borders of destruction site, optimize parameters of ablation, allows us to avoid or minimize undesirable effects, save structures and improve clinical outcomes.

\section{References}

[1] E.S. Tan, B.A. Mulder, M. Rienstra, et al. Pulmonary vein isolation of symptomatic refractory paroxysmal and persistent atrial fibrillation. Neth Heart J. 2009 October; 17(10): 366-372.

[2] Ouyang F, Bansch D, Ernst S, Schaumann A, Hachiya H, Chen M, et al. Complete isolation of left atrium surrounding the pulmonary veins: new insights from the double-Lasso technique in paroxysmal atrial fibrillation. Circulation. 2004;110:2090-6.

[3] Jais P, Hocini M, Hsu LF, Sanders P, Scavee C, Weerasooriya R, et al. Technique and results of linear ablation at the mitral isthmus. Circulation. 2004;110:2996-3002.

[4] Aupperle H, Doll N, Walther T, Ullmann C, Schoon HA, Wilhelm Mohr F. Histological findings induced by different energy sources in experimental atrial ablation in sheep. Interact Cardiovasc Thorac Surg. 2005 Oct;4(5):450-5.

[5] Weber H, Ischinger T. Cardiovascular application of Nd:YAG laser. Laser Med Surg 1988;4:54-8.

[6] Weber H, Enders S, Keiditsch E. Percutaneous Nd:YAG laser coagulation of ventricular myocardium in dogs using a special laser catheter. Pacing Clin Electrophysiol 1989;12:899-910.

[7] Ikeda A, Nakagawa H, Weber H, Sagerer-Gerhardt M, Sharma T, Pitha JV et al. Open-irrigated laser catheter produces deep lesions without thrombus or steam pop. P01-83, Featured Poster Session \& Reception, Heart Rhythm 32nd Annual Scientific Sessions, San Francisco, CA, USA, May 4-7 2011. 